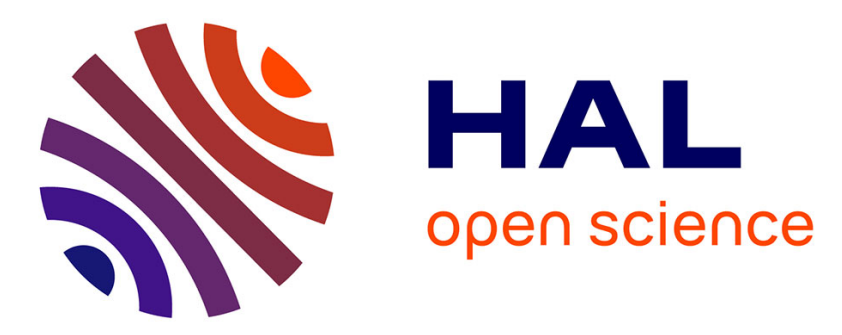

\title{
Crossover in the scale-free statistics of acoustic emission associated with the Portevin-Le Chatelier instability
}

T.A. A Lebedkina, Y. Bougherira, Denis Entemeyer, M.A. Lebyodkin, I.V. V Shashkov

\section{- To cite this version:}

T.A. A Lebedkina, Y. Bougherira, Denis Entemeyer, M.A. Lebyodkin, I.V. V Shashkov. Crossover in the scale-free statistics of acoustic emission associated with the Portevin-Le Chatelier instability. Scripta Materialia, 2018, 148, pp.47-50. 10.1016/j.scriptamat.2018.01.017 . hal-02173968

\section{HAL Id: hal-02173968 \\ https://hal.science/hal-02173968}

Submitted on 11 Jul 2019

HAL is a multi-disciplinary open access archive for the deposit and dissemination of scientific research documents, whether they are published or not. The documents may come from teaching and research institutions in France or abroad, or from public or private research centers.
L'archive ouverte pluridisciplinaire HAL, est destinée au dépôt et à la diffusion de documents scientifiques de niveau recherche, publiés ou non, émanant des établissements d'enseignement et de recherche français ou étrangers, des laboratoires publics ou privés. 


\title{
Crossover in the scale-free statistics of acoustic emission associated with the Portevin-Le Chatelier instability
}

\author{
T.A. Lebedkina ${ }^{\mathrm{a}, \mathrm{b}}$, Y. Bougherira ${ }^{\mathrm{c}}$, D. Entemeyer ${ }^{\mathrm{a}}$, M.A. Lebyodkin ${ }^{\mathrm{a}}$, I.V. Shashkov ${ }^{\mathrm{d}, \mathrm{e}}$ \\ ${ }^{a}$ LEM3 (Laboratoire d'Etude des Microstructures et de Mécanique des Matériaux) \\ CNRS, Université de Lorraine, Arts\&Métiers ParisTech, 7 rue Félix Savart, 57073 Metz, France \\ ${ }^{b}$ Togliatti State University, Belorusskaya St. 14, Tolyatti 445020, Russia \\ ${ }^{c}$ Unité de recherché en optique et photonique (UROP), CDTA, Université de Sétif1, El Bez, Sétif 1900, Algéria \\ ${ }^{d}$ Institute of Solid State Physics RAS, 142432 Chernogolovka, Russia \\ eNational University of Science and Technology "MISIS”, Leninsky Av. 4, 119049 Moscow, Russia
}

\begin{abstract}
The acoustic emission accompanying plastic deformation obeys scale-free statistics reflecting avalanche-like dislocation motion. This feature holds out for the macroscopically unstable deformation of alloys. However, stress serrations display peaked distributions at low enough strain rates. This occurrence of a characteristic macroscopic scale was supposed to result from the synchronization of dislocation avalanches. In the present work, the synchronization mechanism is studied using statistical analysis of different subsets of acoustic events. A crossover in the power-law exponents is detected for the events occurring during deep stress drops. It is described to a transition from chaining to overlapping dislocation avalanches.
\end{abstract}

Keywords: Plastic deformation, Portevin-Le Chatelier effect, Self-organization, acoustic emission.

The Portevin-Le Catelier (PLC) effect is a striking example of self-organization of dislocations giving rise to a macroscopic instability of plastic flow [1]. The phenomenon manifests itself through stress serrations caused by a repetitive abrupt strain localization within deformation bands. Since the 1990s, various approaches to the statistical analysis of jerky flow revealed some characteristic dynamical regimes of plastic deformation on the scale of stress serrations [2-7]. The occurrence of a specific regime was found to depend on the deformation conditions. So, scale-free power-law distributions of serration amplitudes and durations, implying avalanche-like dynamics, are usually observed at high strain rates [2,3,7]. At slower deformation, this behavior was found to change to deterministic chaos that gave rise to complex-shaped histograms [3]. Near-Gaussian histograms corresponding to randomly perturbed relaxation oscillations characterize low strain-rate behavior [7]. Similar and even more complex changes in statistical behavior were observed upon 
temperature variation [8]. It should be noticed for clarity that the relaxation-oscillation instability was predicted in early theories of the PLC effect considering the microscopic mechanism of dynamic strain aging (DSA) of dislocations by solute atoms ant tacitly suggesting that all dislocations perform the same movements $[9,10]$.

Only scale-free statistics was found on the mesoscopic scale relevant to acoustic emission (AE) accompanying plastic deformation in the conditions of the PLC effect [11-13]. This persistence of avalanche-like behavior qualitatively agrees with the predictions of the dynamical model [14-16] developing a unique framework to explain both the PLC serrations and the concomitant AE. Moreover, such a scale invariance was observed for numerous materials in the absence of the PLC effect [17-18], albeit the PLC effect displays a larger range of experimental values of the power-law exponent. The power-law statistics is indicative of a critical dynamics and is often attributed to the phenomenon of self-organized criticality [19,20], notably in the case of smooth plastic flow. An alternative mechanism akin to a turbulent flow at high Reynolds numbers was suggested for the PLC effect [21,22].

The smoothness of the macroscopic deformation curves of most materials bears evidence that the maximum avalanche size is generally confined to mesoscopic length scales most likely related to the microstructure and crystallography of the dislocation glide [23]. Surprisingly, the amplitude of AE events accompanying jerky flow was found to vary in the same range at the instants of stress drops and during smooth loading [11-13]. Therefore, the same limitations should apply to the macroscopically unstable deformation. It is noteworthy that the multifractal analysis [24] rendered similar spectra of generalized dimensions for the series of PLC serrations and the AE signals recorded within the same time interval [11], thus leading to a conjecture of a similar nature of dislocation avalanches during smooth and jerky regimes. To justify these hypotheses and explain the occurrence of intrinsic scales of stress serrations at low and intermediate strain rates, such serrations were supposed to result from synchronization of many dislocation avalanches in the conditions when plastic relaxation during slow reloading result in efficient uniformization of the internal stress field before the next serration. The synchronization phenomena (a striking example is given by synchronous fireflies [25]) refer to repetitive collective movements of either a part or the whole system composed of many coupled elements [26]. The observation of bursts in the duration of $\mathrm{AE}$ events led to a suggestion that the synchronization of dislocations is realized through consecutive triggering (chaining) of dislocation avalanches in neighboring sites, notably, polycrystalline grains. This conjecture agrees with the literature data on the complex nature of individual AE events [27,28] and high-speed video filming of the formation of deformation bands [29]. The possibility of a quasi-simultaneous breakthrough of several avalanches was not explored because of the lack of experimental bases. However, the superposition of the corresponding AE 
events may bias the power-law exponents and prevent from the quantitative description of the PLC instability in terms of the collective dislocation dynamics [30]. In the present work, statistical analysis of various AE subsets is performed with the aim of identifying the mechanisms underlying the AE events in jerky and smooth regimes of the PLC effect and investigating the relative contribution of chaining and superposition of dislocation avalanches during the macroscopic plastic instability.

Details of mechanical testing and AE measurements were described elsewhere [11,12] and will be briefly presented here. Tensile specimens with equiaxed grains $\sim 30 \mu \mathrm{m}$ in size and a gage part of $25 \times 6.8 \times 2.5 \mathrm{~mm}^{3}$ were cut from a cold-rolled sheet of a polycrystalline Al-3\% Mg alloy, annealed at $400^{\circ} \mathrm{C}$ for $2 \mathrm{~h}$, and quenched into water. The tests were performed at room temperature with a constant pulling velocity corresponding to the strain rate $\dot{\varepsilon}_{a}$ from $2 \times 10^{-5} \mathrm{~s}^{-1}$ and $6 \times$ $10^{-3} \mathrm{~s}^{-1}$. The AE was recorded using a piezoelectric transducer fixed to the large specimen end above its gage length. The events detection threshold was set at $U_{0}=27 \mathrm{~dB}$. Conventionally, the $\mathrm{AE}$ event is considered to terminate if starting from this instant the signal remains below $U_{0}$ during the so-called hit definition time (HDT). The HDT was varied between $30 \mu$ s and $300 \mu$ s to check that the results were not influenced by the choice of this parameter within the range commonly used for metals (cf. $[12,13,30]$ ). An additional precaution aimed at filtering out echo signals is that the recording system remains in an idle state during a hit lockout time that was taken equal to HDT. For the sake of comparison with earlier studies [11-13], the statistics was calculated for the squared peak amplitude of $\mathrm{AE}$ hits, $W=A^{2}$, which characterizes the energy dissipated in the deformation process. More precisely, a rescaled variable, $w=W /\langle W\rangle$, was analyzed to facilitate the comparison of histograms for different data subsets. As the objective of the study was to uncover qualitative trends, a direct method based on the calculation of the probability density function (PDF) from the histograms was applied. It was verified (cf. [30,31]) that it renders relatively good estimates of power-law exponents, close to the values obtained by rigorous approaches based on the maximum-likeihood estimation with goodness-of-fit tests [32,33]. The histograms were calculated using equal bin sizes. Since the probability of large events is poor in the case of power-law statistics, the PDF was calculated using the method of variable bin sizes, i.e., the bins with an insufficient number of events were grouped by adding the right-hand neighbours until gathering at least 5 events.

The overall relationship between unstable plastic deformation and $\mathrm{AE}$ was described in detail in [11-13]. It is briefly outlined in Fig. 1 for the low strain rate case characterized by abrupt stress serrations known as type $\mathrm{C}$ behavior [10]. Chart 1(a) shows a portion of the stress-time curve, $\sigma(t)$, recorded at $\dot{\varepsilon}_{a}=2 \times 10^{-5} \mathrm{~s}^{-1}$. The selected interval illustrates the onset of type $\mathrm{C}$ serrations that are caused by brief strain-rate bursts within deformation bands spatially almost uncorrelated 
with each other $[34,35]$. The curve presents three regimes of deformation processes: smooth plastic flow, deep type $\mathrm{C}$ stress drops occurring at a critical strain $\varepsilon_{c r}$ [35], and low-amplitude drops developing before $\varepsilon_{c r}$ but also observed during smooth reloading between deep serrations [36,37]. These small fluctuations are often disregarded in the literature on the PLC effect, but detailed investigations indicate that they are also caused by the DSA mechanism [36]. Like in various materials, the $\mathrm{AE}$ is strong at the beginning of the test, as usually explained by the intense multiplication and long free path of dislocations in the unhardened material $[14,38]$. It diminishes and quickly stabilizes after the elastoplastic transition. Fig. 1 illustrates behavior in such a steady state. Dots in Fig. 1(b) and (c) respectively display series of logarithmic amplitudes $A_{\log }$ and durations $\tau$ of the detected AE hits. Plot 1(d) shows the entire AE signal in a shorter time interval with only two type $\mathrm{C}$ serrations. On the time scale of this plot, noise appears as a black horizontal band, while the signal exceeding the noise level has an intermittent, discrete character, so that individual AE hits correspond to vertical bars. An example of the waveform, corresponding to the hit accompanying the second stress drop, is presented in Fig. 1(e) (more examples can be found in $[13,27,28])$. The totality of data presented in Fig. 1 testify that while amplitudes vary in the same range in all the above-defined regimes, $\tau$ demonstrates bursts at the instants of type $\mathrm{C}$ serrations, thus confirming the hypothesis of clustering (chaining) of dislocation avalanches as the mechanism of formation of the deformation band giving rise to a macroscopic stress drop.
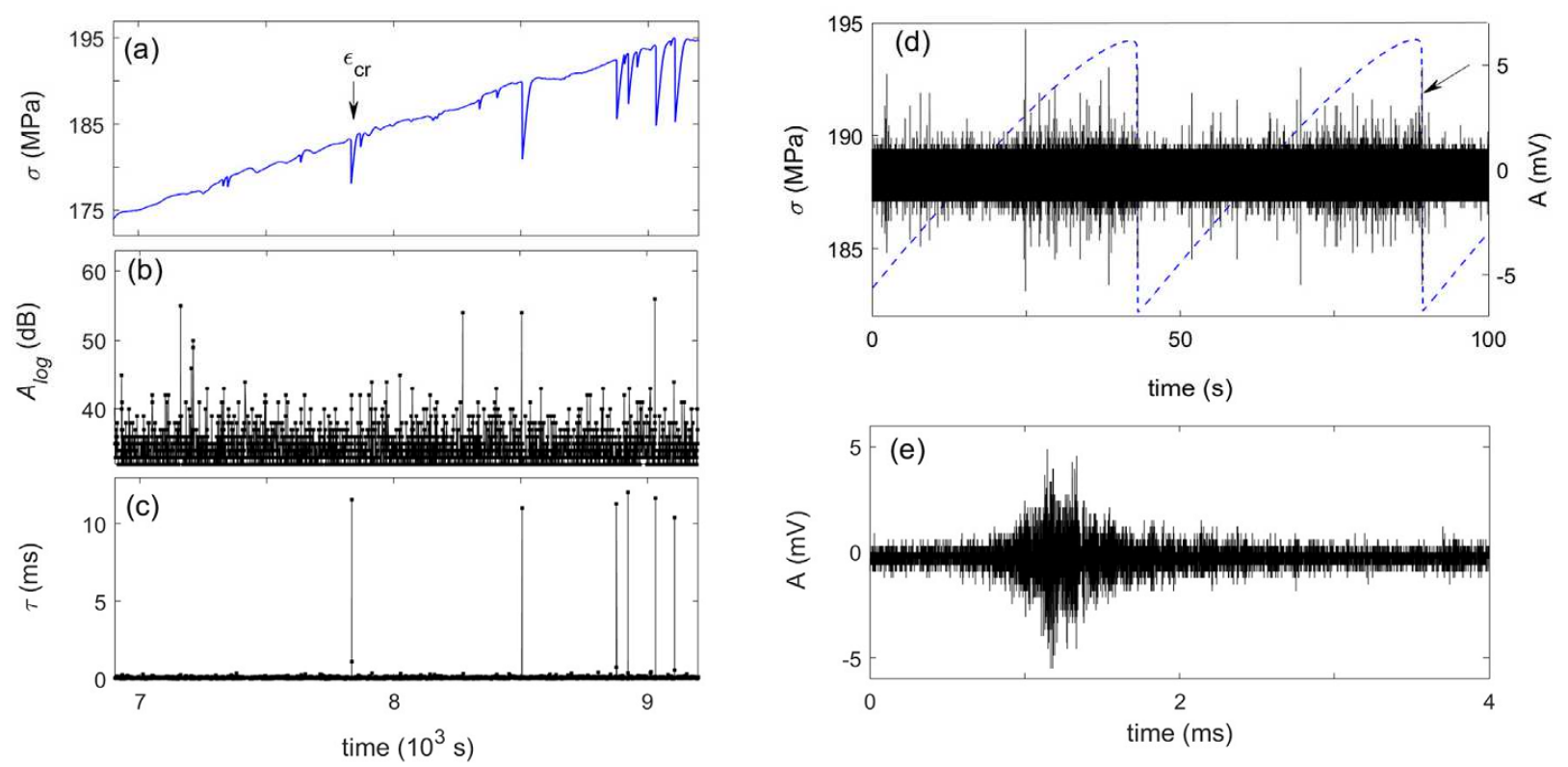

Fig. 1.(a-c) Example of a portion of a stress-time curve $\sigma(t)$ at $\dot{\varepsilon}_{a}=2 \times 10^{-5} \mathrm{~s}^{-1}$ and parameters of the accompanying AE events: the values of logarithmic amplitude $A_{\log }$ and duration $\tau$ are represented by dots (lines drawn as a guide for eye). HDT $=300 \mu \mathrm{s}$. An order of magnitude decrease in the HDT reduces the $\tau$ bursts about twice and does not affect the A range. (d) Comparison of the evolution of $\sigma$ with the as-recorded AE signal. (e) Waveform of the AE hit indicated by an arrow in Chart (d). 
The overall validity of this hypothesis is evidenced by $\tau-A_{\log }$ crossplots representing the entire series of $\mathrm{AE}$ events recorded during a test. In the conditions of type $\mathrm{C}$ behavior illustrated in Fig. 2(a), the events measured after $\varepsilon_{c r}$ (dots) are clearly split into two clouds, the upper of which corresponds to the long-duration hits detected during type $\mathrm{C}$ serrations. Another important observation is that the lower cloud coincides with the events gathered before $\varepsilon_{c r}$ (circles), in agreement with the suggestion of the same nature of dislocation avalanches during smooth and jerky flow. In a more general sense, regardless the PLC effect, they are governed by the same mechanism at different deformation stages, despite the microstructure evolution reflected in the material work hardening. Besides the physical meaning, this observation justifies the application of statistical analysis to various AE subsets.
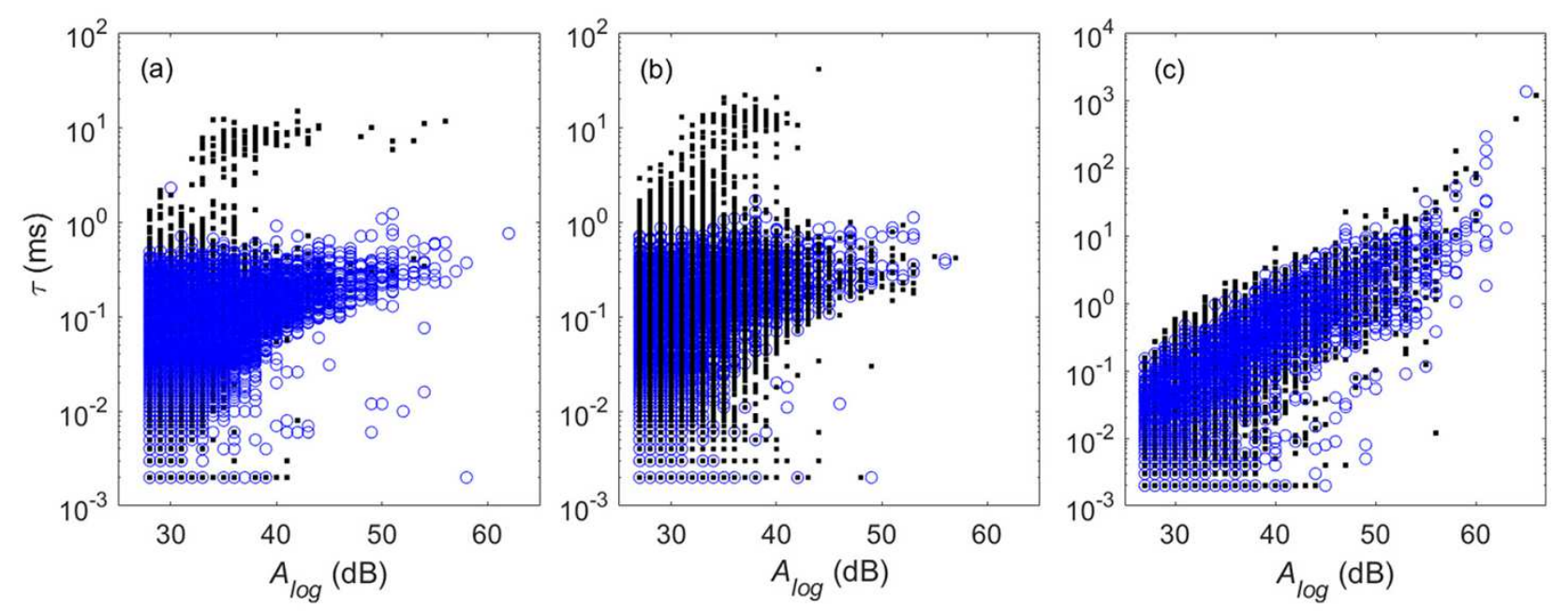

Fig. 2. Examples of $\tau-A_{\log }$ cross-plots for the $\mathrm{AE}$ gathered before (circles) and after (dots) $\varepsilon_{c r}$. (a) $\dot{\varepsilon}_{a}=2 \times$ $10^{-5} \mathrm{~s}^{-1}$, (b) $\dot{\varepsilon}_{a}=2 \times 10^{-4} \mathrm{~s}^{-1}$; (c) $\dot{\varepsilon}_{a}=6 \times 10^{-3} \mathrm{~s}^{-1}$.

When $\dot{\varepsilon}_{a}$ is increased, the corresponding increase in the plastic strain rate is translated through a growing AE activity [12,13]. Consequently, the clustering of AE events is globally enhanced, leading to some increase in the average $\tau$ value. However, the correlation between stress serrations and $\tau$ bursts, which reflects the repetitive clustering caused by the synchronization phenomenon, degrades progressively and becomes indiscernible in the fastest tests. Fig. 2 gives a quantitative meaning to these observations. The separation of two clouds becomes less pronounced at an intermediate $\dot{\varepsilon}_{a}$ in Fig. 2(b) and vanishes at the highest $\dot{\varepsilon}_{a}$ in Fig. 2(c). The latter figure also displays a qualitative change consisting in the occurrence of a clear power-law relationship between $A_{\log }$ and $\tau$. This regime of the PLC effect corresponds to critical type dynamics characterized by power-law statistics of amplitudes and durations of both AE events and stress serrations [2,3,21,22].

This weakening of the correlation between $\tau$ bursts and stress serrations is consistent with the known changes in the stress serration patterns and kinematics of the deformation bands $[2,3,10,34]$. The powerlaw statistics of jerky flow at high strain rates is translated through irregular 
stress fluctuations that cannot be attributed to individual short-duration deformation bands but are associated with quasi continuous propagation of deformation bands along the specimen gage length (type A behavior). The intermediate strain rate range is characterized by transitional patterns (type B behavior) associated with a "relay-race" propagation of deformation bands and regular sequences of stress drops with size varying about a characteristic value. The gradual transition between the three regimes corroborates the continuous character of changes between the $\tau-A_{\log }$ cross-plots and is consistent with theoretical predictions [2,7,14,21,22,39].

Although the totality of data of Figs. 1 and 2 provide evidence of synchronization of dislocation avalanches at low enough strain rates, they do not allow for judging on the specific mechanism of synchronization. Duration bursts testify to an important role of the avalanches chaining. Nevertheless, the stationary range of amplitudes is not sufficient for rejecting the possibility of superposition of AE events from (almost) simultaneous avalanches. To reveal the contribution of such processes, it is necessary to compare the probabilities of different events. Fig. 3 presents examples of such analysis for type $\mathrm{C}$ and type $\mathrm{B}$ conditions. In each case, the series of $\mathrm{AE}$ events was gathered in a time interval corresponding to stabilized jerky flow and AE. Using entire AE sets (dots), power-law PDF, $P(w) \propto w^{-\beta}$, were found for all samples and all deformation conditions (cf. [12,30]). The statistical samples were then subdivided into subsets corresponding to either stress drops (intervals from stress maxima to the subsequent minima) or reloading portions. Moreover, in the type $\mathrm{C}$ case, amplitudes $\Delta \sigma$ of type $\mathrm{C}$ serrations varied about the level of $10 \mathrm{MPa}$ and were clearly separated from the small serrations having amplitudes well below $5 \mathrm{MPa}$. Consequently, it was possible to examine separately the statistics of the AE hits corresponding to two families of stress drops. Fig. 3(a) shows that the PDF obtained for the smooth plastic flow (circles) and small serrations (stars) coincide with the overall dependence. In contrast, the subset gathered during type $\mathrm{C}$ serrations (triangles) reveals more complex behavior. The PDF follows the main dependence, corresponding to $\beta \approx 3.0 \pm 0.1$, in the range of low-amplitude $\mathrm{AE}$ hits ${ }^{1}$. However, a crossover to a shallower power-law dependence with $\beta \approx 2.4 \pm 0.1$ is clearly observed in a range of higher amplitudes (indicated by arrow). It can be concluded that although neither the AE amplitude range nor the avalanche nature of the dislocation dynamics are affected by the macroscopic plastic instability, deep stress serrations are characterized by an increase in the relative probability of high-energy AE events. Moreover, some tendency of this kind features the counterpart curves. Indeed, it should be reminded that the power law statistics observed in many real systems often manifest a cut-off at the large scale of the analyzed variable, generally caused by

\footnotetext{
${ }^{1}$ In the case of the PLC effect, $\beta$ values may depend on the initial microstructure, deformation stage, and strain rate

[12]. Consequently, the present estimates do not have a general meaning.
} 
the finite system size limiting the avalanche size and the finite test duration limiting the available statistics of the rare large events. In contrast, the present data show persistent power-law behavior without any cut-off. This observation concerns even the PDF obtained for smooth plastic flow, thus confirming the uniqueness of the deformation processes during smooth and jerky flow in the aging alloy.

Fig. 3(b) represents similar analysis for type B behavior. In this case, the stress serrations form a single and undivided subset, so that the partial PDF correspond to either smooth plastic flow or stress serrations. The crossover is not observed in this case, in agreement with the suggested weakening of the effect of synchronization with increasing $\dot{\varepsilon}_{a}$. However, the trend to higher probabilities of larger events is confirmed by the absence of a cut-off in the high-amplitude limit. Moreover, the largest AE events manifest an increased probability for jerky flow and, consequently, for the entire set.
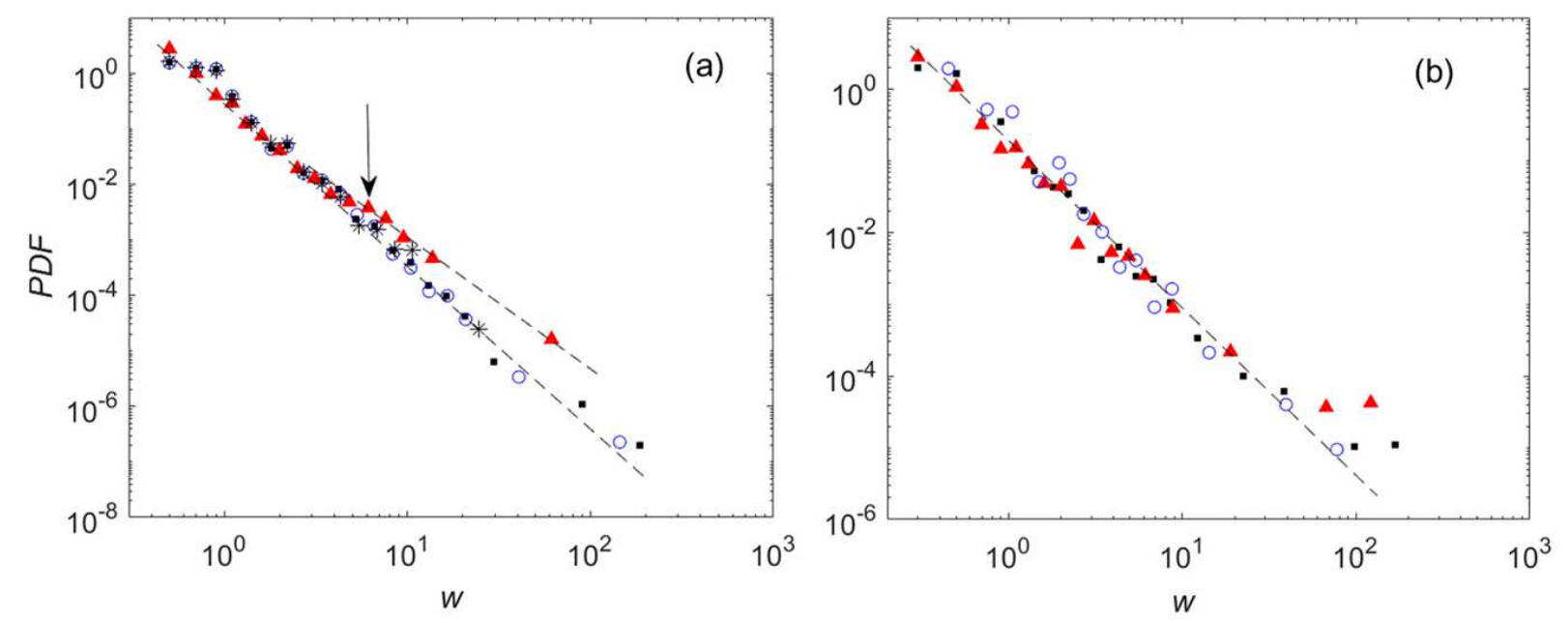

Fig. 3. Examples of PDF of normalized squared amplitudes $w$. for different AE subsets: entire sets (dots), smooth plastic flow (circles), and stress serrations (triangles). In chart (a), triangles designate deep type C drops and stars stand for low-amplitude serrations; arrow indicates the crossover. (a) $\dot{\varepsilon}_{a}=2 \times 10^{-5} \mathrm{~s}^{-1}$, (b) $\dot{\varepsilon}_{a}=2 \times 10^{-4} \mathrm{~s}^{-1}$.

In summary, the data presented allow for the following conclusions:

- The scale-free statistics is found for different subsets of AE events corresponding to either smooth plastic flow or stress serrations, thus testifying that dislocation avalanches are the main deformation processes controlling both smooth and jerky flow of aging alloys.

- Although the power-law exponents are known to depend on the strain rate and the material microstructure [12,13], the statistics of different subsets extracted from the same sample coincide. It can thus be suggested that the elementary avalanches corresponding to smooth and jerky flow are essentially the same.

- Bursts in duration of $\mathrm{AE}$ events at the instants of deep stress serrations at low enough strain rate are likely to be due to consecutive triggering of many dislocation avalanches. Such chaining should 
be warranted by a highly uniform internal stress field formed due to plastic relaxation during slow reloading between stress drops (cf. [12,13]).

- The crossover observed in the statistics of AE events accompanying type $\mathrm{C}$ serrations testifies to an increase in the relative probability of the largest dislocations avalanches. This observation confirms the synchronization hypothesis, on the one hand, and, on the other hand, specifies the approximate character of the model of consecutive triggering. The observed crossover can be explained by (quasi) simultaneous breakthrough of several avalanches due to fast propagation of elastic waves in the material. Nevertheless, since the fraction of such events in the entire statistical sample is low, such a superposition effect does not noticeably bias the overall statistical behavior.

I.Sh. gratefully acknowledges the financial support of the Ministry of Education and Science of the Russian Federation (П02-2017-1-13) in the framework of Increase Competitiveness Program of MISiS, as well funding of a one-month stay at LEM3 by the Center of Excellence "LabEx DAMAS", through the French State program "Investment in the future" (Grant ANR-11-LABX-0008-01 of the French National Research Agency).

\section{References.}

[1] A. Portevin, F. Le Chatelier, C. R. Acad. Sci. 176 (1923) 507.

[2] M.A. Lebyodkin, Y. Brechet, Y. Estrin, L.P. Kubin, Phys. Rev. Lett. 74 (1995) 4758.

[3] M.S. Bharathi, M. Lebyodkin, G. Ananthakrishna, C. Fressengeas, L.P. Kubin, Acta Mater. 50 (2002) 2813.

[4] A. Sarkar, C.L. Webber Jr, P. Barat, P. Mukherjee, Phys. Lett. A 372 (2008) 1101.

[5] A.C. Iliopoulos, N.S. Nikolaidis, E.C. Aifantis, Phys. A 438 (2015) 509.

[6] K. Darowicki, J. Orlikowski, A. Zieliński, W. Jurczak, Comput. Mater. Sci. 39 (2007) 880.

[7] M.A. Lebyodkin, L.R. Dunin-Barkowskii, Y. Brechet, Y. Estrin, L.P. Kubin, Acta Mater. 44 (2000) 2529.

[8] M.A. Lebedkin, L.R. Dunin-Barkovskii, Phys. Solid State 40 (1998) 447.

[9] P. Penning, Acta Metall. 20 (1972) 1169.

[10] Y. Estrin, L.P. Kubin, in: H.-B. Mühlhaus (Ed.), Continuum Models for Materials with Microstructure, John Wiley\& Sons Ltd. 1995, pp. 395-450. 
[11] M.A. Lebyodkin, N.P. Kobelev, Y. Bougherira, D. Entemeyer, C. Fressengeas, T.A. Lebedkina, I.V. Shashkov, Acta Mater. 60 (2012) 844.

[12] M.A. Lebyodkin, N.P. Kobelev, Y. Bougherira, D. Entemeyer, C. Fressengeas, V.S. Gornakov, T.A. Lebedkina, I.V. Shashkov, Acta Mater. 60 (2012) 3729.

[13] I.V. Shashkov, M.A. Lebyodkin, T.A. Lebedkina, Acta Mater. 60 (2012) 6842.

[14] R. Sarmah, G. Ananthakrishna, Acta Mater. 91 (2015) 192.

[15] J. Kumar, R. Sarmah, G. Ananthakrishna, Phys. Rev. E 92 (2015) 144109.

[16] J. Kumar, G. Ananthakrishna, Phys. Rev. E 97 (2018) 012201.

[17] M.C. Miguel, A. Vespignani, S. Zapperi, J. Weiss, J.R. Grasso, Nature 410 (2001) 667.

[18] M. Zaiser, Adv. Phys. 55 (2007) 185.

[19] P. Bak, C. Tang, K. Wiesenfeld, Phys. Rev. A 38 (1988) 364.

[20] D.L. Turcotte, Rep. Prog. Phys. 62 (1999) 1377.

[21] M.S. Bharathi, G. Ananthakrishna, Europhys. Lett. 60 (2002) 234.

[22] G. Ananthakrishna, M.S. Bharathi, Phys. Rev. E 70 (2004) 026111.

[23] J. Weiss, T. Richeton, F. Louchet, F. Chmelık, P. Dobron, D. Entemeyer, M. Lebyodkin, T. Lebedkina, C. Fressengeas, R.J. McDonald, Phys. Rev. B 76 (2007) 224110.

[24] T.C. Halsey, M.H. Jensen, L.P. Kadanoff, I. Procaccia, B.I. Shraiman, Phys. Rev. A 33 (1986) 1141.

[25] J. Buck, E. Buck, Sci. Am. 234 (1976) 74.

[26] S.H. Strogatz, Phys. D 143 (2000) 1.

[27] M.A. Lebyodkin, T.A. Lebedkina, F. Chmelik, T.T. Lamark, Y. Estrin, C. Fressengeas, J. Weiss, Phys. Rev. B 79 (2009) 174114.

[28] A. Vinogradov, A. Lazarev, Scr. Mater. 66 (2012) 745.

[29] A.A. Shibkov, A.E. Zolotov, M.A. Zheltov, A.A. Denisov, Crystallogr. Rep. 57 (2012) 105.

[30] M.A. Lebyodkin, I.V. Shashkov, T.A. Lebedkina, K. Mathis, P. Dobron, F. Chmelik, Phys. Rev. E 88 (2013) 042402. 
[31] M.A. Lebyodkin, I.V. Shashkov, T.A. Lebedkina, V.S. Gornakov, Phys. Rev. E 95 (2017) 032910.

[32] A. Clauset, C. Shalizi, M. Newman, SIAM Rev. 51 (2009) 661.

[33] A. Deluca, Á. Corral, Acta Geophys. 61 (2013) 1351.

[34] H. Jiang, Q. Zhang, X. Chen, Z. Chen, Z. Jiang, X. Wu, J. Fan, Acta Mater. 55 (2007) 2219.

[35] M.A. Lebyodkin, T.A. Lebedkina, Phys. Rev. E 77 (2008) 026111.

[36] N.P. Kobelev, M.A. Lebyodkin, T.A. Lebedkina, Metall. Mater. Trans. A 48A (2017) 965.

[37] M. Abbadi, P. Hähner, A. Zeghloul, Mater. Sci. Eng. A 337 (2002) 194.

[38] K. Mathis, F. Chmelik, in: W. Sikorsky (Ed.), Acoustic Emission, InTech, Rijeka 2012, pp. 23-48.

[39] S. Kok, M.S. Bharathi, A.J. Beaudoin, C. Fressengeas, G. Ananthakrishna, L.P. Kubin, M. Lebyodkin, Acta Mater. 51 (2003) 3651 